\title{
The impact of lockdown during the COVID-19 pandemic on mental and social health of children and adolescents
}

\author{
Michiel A. J. Luijten ${ }^{1,2} \cdot$ Maud M. van Muilekom ${ }^{1} \cdot$ Lorynn Teela $^{1} \cdot$ Tinca J. C. Polderman ${ }^{3} \cdot$ Caroline B. Terwee $^{2}$. \\ Josjan Zijlmans ${ }^{3} \cdot$ Leonie Klaufus $^{4,5} \cdot$ Arne Popma $^{1,3} \cdot$ Kim J. Oostrom $^{1} \cdot$ Hedy A. van Oers ${ }^{1} \cdot$ Lotte Haverman $^{1}$ (1)
}

Accepted: 23 April 2021 / Published online: 15 May 2021

(c) The Author(s) 2021

\begin{abstract}
Purpose During the COVID-19 pandemic in the Netherlands, governmental regulations resulted in a lockdown for adults as well as children/adolescents. Schools were closed and contact with other people was limited. In this cross-sectional, population-based study, we aimed to investigate the mental/social health of children/adolescents during COVID-19 lockdown. Methods Two representative samples of Dutch children/adolescents (8-18 years) before COVID-19 $(2018, N=2401)$ and during lockdown (April 2020, $N=844$ ) were compared on the Patient-Reported Outcomes Measurement Information System (PROMIS) domains: global health, peer relationships, anxiety, depressive symptoms, anger, sleep-related impairment by linear mixed models and calculating relative risks (RR $(95 \% \mathrm{CI})$ ) for the proportion of severe scores. Variables associated with worse mental/social health during COVID-19 were explored through multivariable regression models. The impact of COVID-19 regulations on the daily life of children was qualitatively analyzed.

Results Participants reported worse PROMIS T-scores on all domains during COVID-19 lockdown compared to before (absolute mean difference range 2.1-7.1 (95\% CI 1.3-7.9). During lockdown, more children reported severe Anxiety (RR $=1.95$ (1.55-2.46) and Sleep-Related Impairment $(\mathrm{RR}=1.89$ (1.29-2.78) and fewer children reported poor Global Health $(\mathrm{RR}=0.36$ $(0.20-0.65))$. Associated factors with worse mental/social health were single-parent family, $\geq$ three children in the family, negative change in work situation of parents due to COVID-19 regulations, and a relative/friend infected with COVID-19. A large majority ( $>90 \%$ ) reported a negative impact of the COVID-19 regulations on daily life.

Conclusion This study showed that governmental regulations regarding lockdown pose a serious mental/social health threat on children/adolescents that should be brought to the forefront of political decision-making and mental healthcare policy, intervention, and prevention.
\end{abstract}

Michiel A. J. Luijten and Maud M. van Muilekom contributed equally to this study.

Lotte Haverman

1.haverman@amsterdamumc.nl

1 Child and Adolescent Psychiatry \& Psychosocial Care, Amsterdam Reproduction and Development, Amsterdam Public Health, Emma Children's Hospital, Amsterdam UMC, University of Amsterdam, G8-136, Meibergdreef 9, Postbox 22660, 1100 DD Amsterdam, The Netherlands

2 Epidemiology and Data Science, Vrije Universiteit, Amsterdam UMC, Amsterdam, The Netherlands

3 Child and Adolescent Psychiatry \& Psychosocial Care, Amsterdam Public Health, Vrije Universiteit Amsterdam, Amsterdam UMC, De Boelelaan 1117, Amsterdam, The Netherlands
4 Department of Public and Occupational Health, Vrije Universiteit Amsterdam, Amsterdam UMC, Amsterdam, The Netherlands

5 Department of Epidemiology, Health Promotion, and Health Care Innovation, Public Health Service Amsterdam, Amsterdam, The Netherlands 


\section{Introduction}

The COVID-19 pandemic has an enormous impact on society as a whole, and on children and adolescents in particular. Although children and adolescents are less affected by morbidity and mortality [1], the restrictions imposed by governments worldwide profoundly impact their daily life, including their mental and social health [2].

In the Netherlands, the first COVID-19 patient was identified on February 27th 2020 and restrictions were imposed by the government starting on March 12th 2020. People were asked to stay inside and work from home as much as possible, to comply with social distancing $(1.5 \mathrm{~m})$, and all large events were canceled. On March 15th, a 'partial' lockdown was implemented (www.gover nment.nl/topics/coronavirus-covid-19). All schools and child care facilities were closed (except for children whose parents had an occupation classified as essential), as well as sports and leisure facilities, bars, and restaurants. However, children were still allowed to play outside, and visitors up to three persons were permitted at home. On May 11 th primary schools were partially reopened and on June 2nd secondary schools followed.

During the lockdown, children and adolescents were experiencing physical isolation from their classmates, friends, teachers, and other important adults (e.g., grandparents). This might not only result in feelings of loneliness but could potentially lead to precarious situations for children from unsafe domestic situations, due to a lack of escape possibilities. In addition, children and adolescents may experience mental health problems due to the COVID-19 pandemic itself, such as increased anxiety, as they might fear that they or their loved ones will get infected or they might worry about the future of the world.

Several cross-sectional and longitudinal studies on the effects of the COVID-19 pandemic on mental health in adults have now been published. Increased levels of anxiety, depression, suicidal ideation, and (post-traumatic) stress, decreased psychological well-being, and a high percentage of sleep problems have been reported [3-10]. Poor mental health was associated with female gender, younger age, low educational level, living alone/being divorced, having no work, low income, a socially disadvantaged background, and having an infected relative with COVID19 [3-10].

However, studies on the effects of the COVID-19 lockdown on the social and mental health of children and adolescents are yet sparse. Two reviews reflect on the possible effects of social isolation, and they have painted an image of loneliness, anxiety, and depression [11, 12]. Additionally, current opinion papers regarding the COVID-19 outbreak have described increased tension at home and child abuse as possible consequences during the lockdown [13, 14]. Several authors fear that the lockdown will magnify existing health disparities and that certain communities (e.g., with migrant background and low socioeconomic status) will be more vulnerable to develop mental and social health issues $[12,15,16]$.

One systematic review on the effects of previous pandemics on the mental health of children and adolescents is available, indicating that social isolation and quarantining have a negative impact on anxiety, depressive, and fear symptoms [2]. Additionally, three cross-sectional survey studies are currently available from China, focusing on the impact on the mental health of the COVID-19 pandemic and lockdown specifically. They reported prevalences of anxiety and depressive symptoms of $19 \%$ and $23 \%$ respectively for primary school children [17], of $37 \%$ and $44 \%$ respectively for high school children [18], and clinically elevated depressive symptoms scores for $>22 \%$ of children and adolescents [19] during the COVID-19 lockdown. Especially girls, older children, children living in an urban region, and children having a COVID-19 infected friend/relative appeared to be most prone to mental health problems [18, 19]. The reported prevalences were higher compared to pre-COVID-19 established cut-offs and percentages in China, however, none of these differences were statistically tested. Recently published studies have shown that children did report statistically significant lower health-related quality of life and higher anxiety and depression levels than before the pandemic [20-23].

A better understanding of how the governmental restrictions during the COVID-19 pandemic affect children's and adolescents' mental and social health can help guide future interventions and inform policymakers. The current study compares the mental and social health of a representative sample of Dutch children and adolescents during the COVID-19 lockdown to earlier collected before COVID19 reference data. The aims of this study were to: (1) quantify differences in mental and social health of children and adolescents before and during the COVID-19 lockdown, (2) identify factors that are associated with poorer mental and social health during the COVID-19 lockdown, (3) examine the change in overall atmosphere at home before and during the COVID-19 lockdown, and (4) qualitatively assess the impact of the COVID-19 lockdown on the daily life of children and adolescents. 


\section{Methods}

\section{Participants and procedure}

\section{Before COVID-19}

As part of a larger Patient-Reported Outcome Measurement Information System (PROMIS) validation study, two studies were conducted between December 2017 and July 2018 in the Dutch general population to collect representative data of children and adolescents (8-18 years) on physical, mental, and social aspects of health [24][Klaufus et al., submitted article]. A two-step random stratified sampling method was used to ensure representativeness on key demographics. Parents participating in existing panels were approached by two independent online research agencies ('Kantar Public' or 'Panel Inzicht'). Both panels consist of families living across the Netherlands, that provided informed consent to be approached through e-mail for completing questionnaires for a small financial compensation. Children were subsequently approached by their parents to complete self-report questionnaires. Children completed the questionnaires through our research website of the KLIK Patient-Reported Outcome Measures (PROM) portal [25] or the panel website. Parents were asked to complete a socio-demographic questionnaire. All children and parents provided informed consent and the studies were approved by the Medical Ethics Committee Amsterdam UMC. The samples were representative of the Dutch general population within $2.5 \%$ on most key demographics (age, gender, ethnicity, region, and educational level) compared to population numbers in 2017 (Gold Standard - Statistics Netherlands, www.cbs.nl/en-gb) [24]. Marital status and parental educational level data were differently categorized in the before COVID-19 data collection of the PROMIS Anxiety and Depressive Symptoms domains and therefore not usable for this study [Klaufus et al., submitted article].

\section{During COVID-19}

During the COVID-19 lockdown, between April 10th and May 5th 2020, data were collected by 'Panel Inzicht' from another sample of children and adolescents from the Dutch general population. The aim was to collect data on the same measures in a representative sample of approximately 1000 children with similar characteristics (within $2.5 \%$ of the previously mentioned key demographics) as the before COVID-19 sample. Data collection procedures were similar as in 2018, with the addition of a few COVID-19 specific questions for children, adolescents and parents. Children and parents provided informed consent and the study was approved by the Medical Ethics Committee Amsterdam UMC.

\section{Measures}

\section{Socio-demographic questionnaires}

Parents completed questions about themselves (region of residence, country of birth, educational level, marital status, and number of children) and their child (age, gender).

\section{PROMIS pediatric measures}

PROMIS item banks and scales were developed and validated, to measure generic unidimensional domains (e.g., anxiety) of physical, social or mental health using modern psychometric techniques. The item banks can be administered as Computerized Adaptive Test (CAT), where items are selected based on responses to previously completed items, resulting in a reliable score with a few items [26]. Six Dutch-Flemish PROMIS pediatric measures (Scale V2.0-Anger [27], CAT V2.0-Peer Relationships [28], Scale V1.0-Global health $(7+2)$ [29], CAT V1.0-Sleeprelated Impairment [30], CAT V2.0-Anxiety [31], and CAT V2.0-Depressive Symptoms [31]) were completed by children and adolescents. These measures have been selected by the American Psychiatric Association (APA) as level-2 assessment measures for monitoring and evaluating psychiatric disorders from the DSM-5. All PROMIS measures use a 7-day recall period, and items are scored on a fivepoint Likert scale. All items range from 'never' to '(almost) always' except for Global Health, where response categories differ for each item (e.g. 'excellent' to 'poor'). Total scores are calculated by transforming the item scores into a $T$-score which has a mean of 50 and standard deviation (SD) of 10 in the U.S. general population. For all measures, higher scores represent more of the construct. For Anger ( 9 items) and Global Health $(7+2$ items $)$ all items were administered. The US item parameters were used in the CAT algorithm and $T$-score calculations, as by PROMIS convention.

\section{COVID-19-related additional questions}

Three closed-ended questions were added for parents about whether there was a negative change in work situation of one of the parents/caregivers due to COVID-19 regulations (e.g., loss of income, reduced number of working hours, unemployment), whether a friend or relative had been infected with COVID-19 and if the child still attended child care/school during lockdown (e.g., both parents performing essential occupations). 
Children and adolescents were asked to complete three COVID-19-specific questions: 'How did you experience the atmosphere at home before the schools were closed?' and 'How do you experience the atmosphere at home now?' rated on a visual analogue scale (VAS) ranging from 0 'Not pleasant at all' to 100 'Very pleasant', and an open-ended question 'How are the corona-regulations for you?'.

\section{Statistical analyses}

For all statistical analyses the Statistical Package for Social Sciences (SPSS), version 26.0, was used.

First, descriptive analyses (mean and percentages) were used to characterize the participants in the different samples. To compare samples, independent T-tests (for continuous variables) or chi-square tests of independence (for categorical variables) were performed. Effect sizes were calculated for continuous variables (Cohen's $d$ ) and categorical variables (risk ratio or Cramer's $V$ ).

Second, to test whether mental and social health of the sample during COVID-19 differed from the sample before COVID-19 on PROMIS $T$-scores, a one-way analysis of covariance (ANCOVA) was performed per PROMIS domain, adjusted for differences in sociodemographic characteristics. Mean differences (95\% CI) were reported.

Percentages of children and adolescents reporting 'severe' symptoms or 'poor' functioning on the PROMIS measures before and during the COVID-19 lockdown were compared. Severe symptoms or poor functioning was defined as a $T$-score $1.5 \mathrm{SD}$ above or below the mean $T$-score before COVID-19 respectively, except for Peer Relationships, where $2 \mathrm{SD}$ was used as cut-off for poor functioning (see www.healthmeasures.net). Differences in proportions of severe scores were tested using chi-squares tests of independence and the relative risk (RR) with $95 \%$ confidence intervals (95\% CI) were reported. The RR represents the risk of a child measured during the COVID-19 lockdown having a severe score compared to before COVID-19. A ratio $>1$ indicates more risk.

Third, to determine which variables were significantly associated with mental and social health during the COVID19 lockdown, a multivariable linear regression analysis was performed for each PROMIS domain. The following variables were included as independent variables: age, gender, parental country of birth, marital status, region, number of children in the family, parental educational level, change in work situation due to COVID-19 regulations, infected relative/friend with COVID-19 and if the child still attended child care/school during lockdown. No multicollinearity was present between these variables (all correlations $<0.50$ ). Per domain the effect size of all independent variables was reported, expressed as unstandardized regression coefficient $B(95 \% \mathrm{CI})$.
Fourth, to investigate changes in atmosphere at home a paired T-test was used to test the difference between the two single items. Mean difference (95\% CI) was reported.

Fifth, to assess the impact of the COVID-19 lockdown on the daily life of children, the open-ended question 'How are the corona-regulations for you?' was qualitatively analyzed (by LT and HAvO) using thematic analysis [32]. The answers were categorized into positive, neutral or negative experiences and thereafter clustered into themes. Themes were ranked according to their frequency of occurrence (high to low).

\section{Results}

\section{Sociodemographic characteristics}

During the COVID-19 lockdown, 844 children participated. This sample showed similar characteristics (most variables within $2.5 \%$ from each other) compared to the sample collected before COVID-19 (total $N=2401$ ). Significant differences were found on four variables (Table 1); age (during $\mathrm{M}=13.4(\mathrm{SD}=2.80)$ versus before $\mathrm{M}=13.1(\mathrm{SD}=3.14)$, mean difference $=0.3 ; 95 \%$ CI -0.54 to $-0.06, d=0.10$ ), at least one parent born in a foreign country (during $11.8 \%$ versus before $20.2 \%, \chi^{2}(1)=29.884, P<0.001, \mathrm{RR}=0.58$ ), parents with a low educational level (during $9.0 \%$ versus before $12.8 \%, \chi^{2}(2)=7.470, P=0.024$, Cramer's $V=0.06$ ), and families with one child (during $25.5 \%$ versus before $15.5 \%, \chi^{2}(2)=44.728, P<0.001$, Cramer's $V=0.12$ ).

\section{Differences in mental and social health in children and adolescents during versus before the COVID-19 lockdown}

During the COVID-19 lockdown, children and adolescents reported worse $T$-scores than children and adolescents before the COVID-19 lockdown on all PROMIS domains, after controlling for age, parental country of birth, parental educational level and the number of children (absolute mean difference range, 2.06-7.05; absolute 95\% CI range, 1.25-7.86) (Table 2). Largest differences were found for Anxiety (mean difference $=7.1,95 \%$ CI 6.2-7.9) and Depressive Symptoms (mean difference $=4.9 ; 95 \%$ CI 4.0-5.7).

Significantly more children reported severe Anxiety (during $16.7 \%$ versus before $8.6 \%$; RR, 1.95 ; 95\% CI 1.55-2.46) and severe Sleep-Related Impairment (during $11.5 \%$ versus before $6.1 \%$; RR, 1.89 ; 95\% CI 1.29-2.78) during the COVID-19 lockdown than before COVID-19 (Table 3). Fewer children reported poor Global Health (during 1.7\% versus before $4.6 \%$; RR, 0.36 ; 95\% CI 0.20-0.65). 
Table 1 Socio-demographic characteristics of participants per group

\begin{tabular}{|c|c|c|}
\hline & General population sample during COVID-19 & General population sample before COVID-19 \\
\hline$N^{\mathrm{a}}$ & 844 & 2401 \\
\hline \multirow[t]{2}{*}{ Mean age in years $(\mathrm{SD})^{*}$} & $13.4(2.8)$ & $13.1(3.1)$ \\
\hline & $\%$ & $\%$ \\
\hline Gender (male) & 47.4 & 50.3 \\
\hline \multicolumn{3}{|l|}{ Region $^{\mathrm{b}}$} \\
\hline North & 12.6 & 10.6 \\
\hline East & 22.2 & 23.8 \\
\hline South & 25.0 & 23.2 \\
\hline West & 40.3 & 42.4 \\
\hline \multicolumn{3}{|l|}{ Number of children in family* } \\
\hline 1 Child & 25.5 & 15.5 \\
\hline 2 Children & 46.6 & 49.8 \\
\hline 3 Children or more & 27.9 & 34.7 \\
\hline \multicolumn{3}{|l|}{ Country of birth parents* } \\
\hline Both parents Netherlands & 88.2 & 79.8 \\
\hline At least one parent in foreign country & 11.8 & 20.2 \\
\hline Marital status parents & & $\mathrm{c}$ \\
\hline Two parent family & 82.0 & 84.7 \\
\hline Single parent family & 18.0 & 15.3 \\
\hline Educational level parents*d & & $\mathrm{c}$ \\
\hline Low & 9.0 & 12.8 \\
\hline Intermediate & 51.8 & 48.2 \\
\hline High & 39.2 & 38.9 \\
\hline \multicolumn{3}{|l|}{ Corona-specific variables } \\
\hline Infected relative/friend (yes) & 23.7 & . \\
\hline Negative change in work situation (yes) & 26.2 & . \\
\hline Daycare/school attendance child (yes) & 5.5 & . \\
\hline
\end{tabular}

\section{$S D$ standard deviation}

*Significant difference between the two samples with $P<0.05$

${ }^{a}$ Due to missing values, number of respondents vary across different socio-demographic variables

${ }^{\mathrm{b}}$ Region: North=Groningen, Friesland, Drenthe, East=Overijssel, Gelderland, Flevoland, South=Zeeland, Noord-Brabant, Limburg, West $=$ Utrecht, Noord-Holland, Zuid-Holland

${ }^{\mathrm{c}}$ Based on $N=1082$ due to missing values of these variables for the PROMIS Anxiety and Depressive Symptoms measures

${ }^{\mathrm{d}}$ Educational level parents, Low = primary, lower vocational education, lower and middle general secondary education; Intermediate $=$ middle vocational education, higher secondary education, pre-university education; High = higher vocational education, university

\section{Variables associated with poor mental and social health in children and adolescents during the COVID-19 lockdown}

Lower Global Health was associated with a single-parent family ( $B=-3.00 ; 95 \%$ CI -4.23 to -1.76$)$. Lower Peer Relationships were reported by boys compared to girls $(B=-1.25 ; 95 \%$ CI -2.23 to -0.27$)$. Increased Anxiety was associated with age $(B=-0.34 ; 95 \%$ CI -0.53 to -0.15$)$, a single-parent family $(B=1.46 ; 95 \%$ CI $0.11-2.81)$, an infected relative or friend $(B=1.94 ; 95 \%$ CI $0.72-3.16)$, and parents with a negative change in a work situation
( $B=3.01 ; 95 \%$ CI 1.84-4.18). More depressive symptoms were associated with highly educated parents (where intermediate differed from lower; $B=2.24 ; 95 \%$ CI $0.23-4.24$ ) and parents with a negative change in a work situation ( $B=2.45$; 95\% CI $1.20-3.70)$. More Anger was associated with age ( $B=-0.47 ; 95 \%$ CI -0.67 to -0.28$)$, highly educated parents (where intermediate differed from lower; $B=2.30,95 \%$ CI $0.27-4.33$ and high differed from lower; $B=2.10$; 95\% CI $0.01-4.19)$, three or more children $(B=2.07 ; 95 \%$ CI $0.52-3.62)$ and parents with a negative change in a work situation $(B=1.71 ; 95 \%$ CI $0.45-2.98)$. Finally, more Sleep-Related Impairment was related to the 
Table 2 Mean PROMIS T-scores and significant mean differences in the general population during and before COVID-19, adjusted for age, parental country of birth, parental educational level and number of children

\begin{tabular}{|c|c|c|c|c|c|c|c|c|c|}
\hline & \multicolumn{3}{|c|}{$\begin{array}{l}\text { General population sample } \\
\text { during COVID-19 }\end{array}$} & \multicolumn{3}{|c|}{$\begin{array}{l}\text { General population sample } \\
\text { before COVID-19 }\end{array}$} & \multirow[t]{2}{*}{$P^{\mathrm{a}}$} & \multirow[t]{2}{*}{$\eta^{2}$} & \multirow[t]{2}{*}{ Mean difference $(95 \% \mathrm{CI})^{\mathrm{b}}$} \\
\hline & $N$ & M & SD & $N$ & M & SD & & & \\
\hline PROMIS Global Health ${ }^{\mathbf{c}}$ & 813 & 46.2 & 6.9 & 1082 & 48.3 & 9.8 & $<0.01$ & 0.01 & $-2.1(-2.9$ to -1.3$)$ \\
\hline PROMIS Peer Relationships ${ }^{\mathbf{c}}$ & 813 & 44.3 & 7.0 & 527 & 46.9 & 9.5 & $<0.01$ & 0.02 & $-2.6(-3.5$ to -1.7$)$ \\
\hline PROMIS Anxiety ${ }^{\mathrm{d}}$ & 813 & 50.5 & 7.6 & 1318 & 43.8 & 9.7 & $<0.01$ & 0.12 & $7.1(6.2 \text { to } 7.9)^{\mathrm{e}}$ \\
\hline PROMIS Depressive Symptoms ${ }^{\mathrm{d}}$ & 813 & 49.4 & 8.0 & 1318 & 44.7 & 10.6 & $<0.01$ & 0.05 & $4.9(4.0 \text { to } 5.7)^{\mathrm{e}}$ \\
\hline PROMIS Anger ${ }^{d}$ & 813 & 47.3 & 8.2 & 527 & 44.2 & 11.4 & $<0.01$ & 0.02 & $3.1(2.0$ to 4.1$)$ \\
\hline PROMIS Sleep Related Impairment ${ }^{\mathrm{d}}$ & 813 & 49.9 & 8.7 & 527 & 47.6 & 10.0 & $<0.01$ & 0.02 & $2.5(1.5$ to 3.5$)$ \\
\hline
\end{tabular}

$\eta^{2}$ Amount of variance explained by group membership, $C I$ confidence interval, $M$ mean, $S D$ standard deviation

${ }^{\text {a }} P$-value of the main effect of the ANCOVA

${ }^{\mathrm{b}}$ Adjusted mean differences and CI

${ }^{\mathrm{c}}$ Higher scores indicate better functioning

${ }^{\mathrm{d}}$ Higher scores indicate more symptoms

${ }^{\mathrm{e}}$ Not corrected for parental educational level due to missing values

Table 3 Percentage of participants with poor functioning or severe symptoms (>1.5 SD) on the PROMIS domains for both samples

\begin{tabular}{|c|c|c|c|}
\hline & $\begin{array}{l}\text { General population sample during } \\
\text { COVID-19 } \\
\%\end{array}$ & $\begin{array}{l}\text { General population sample before } \\
\text { COVID-19 } \\
\%\end{array}$ & Relative risk $(95 \% \mathrm{CI})$ \\
\hline PROMIS Global Health* & 1.7 & 4.6 & $0.36(0.20-0.65)$ \\
\hline PROMIS Peer Relationships ${ }^{\mathrm{a}}$ & 1.9 & 1.9 & $0.99(0.46-2.19)$ \\
\hline PROMIS Anxiety* & 16.7 & 8.6 & $1.95(1.55-2.45)$ \\
\hline PROMIS Depressive Symptoms & 7.1 & 8.2 & $0.87(0.64-1.18)$ \\
\hline PROMIS Anger & 3.7 & 5.5 & $0.67(0.41-1.09)$ \\
\hline PROMIS Sleep-Related Impairment* & 11.5 & 6.1 & $1.89(1.29-2.78)$ \\
\hline
\end{tabular}

CIConfidence interval

*Significant difference between during and before with $P$ value $<0.01$

${ }^{a}$ For Peer Relationships a cut-off of 2 SD was used, as per HealthMeasures guidelines.

country of birth of parents ( $\geq$ one foreign parent; $B=1.95$; 95\% CI 0.13-3.77), a single-parent family ( $B=2.07$; $95 \%$ CI 0.53-3.62) and parents with a negative change in a work situation ( $B=2.53 ; 95 \%$ CI 1.19-3.87) (Table 4).

\section{Changes in atmosphere at home during the COVID-19 lockdown}

Children and adolescents reported a worse atmosphere (mean difference $=-3.1 ; 95 \% \mathrm{CI}-4.1$ to -2.1 ) at home during the COVID-19 lockdown $(M=78.2, \mathrm{SD}=17.9)$ than before COVID-19 $(\mathrm{M}=81.4, \mathrm{SD}=16.0)$.

\section{Impact of COVID-19 regulations on the daily life of children}

The majority $(\sim 90 \%)$ of children indicated that the COVID-19 lockdown had a negative impact on their daily life. The most often mentioned issues (>50 children) were: (1) missing contact with friends, (2) not allowed to go to school, (3) missing freedom, (4) not allowed to participate in sports, (5) missing joyful activities (e.g., birthdays, holidays, parties, shopping), (6) difficulties with homeschooling (7) missing extended family, and (8) boredom (Table 5). A minority of children did not experience any difficulties with the COVID-19 lockdown regulations $(\sim 7 \%)$ (e.g., 'It does not bother me') or reported positive consequences $(\sim 3 \%)$ (e.g., 'I really like that I can play with children in my neighborhood all day long'). 
Table 4 Variables associated with mental and social health in children and adolescents during the COVID-19 lockdown

\begin{tabular}{|c|c|c|c|c|c|c|}
\hline & Global Health $^{\mathrm{a}}$ & Peer Relationships ${ }^{\mathrm{a}}$ & Anxiety ${ }^{b}$ & $\begin{array}{l}\text { Depressive } \\
\text { Symptoms }\end{array}$ & Anger $^{b}$ & $\begin{array}{l}\text { Sleep-Related } \\
\text { Impairment }{ }^{b}\end{array}$ \\
\hline Covariates & $B$ & $B$ & $B$ & $B$ & $B$ & $B$ \\
\hline Age & -0.03 & 0.11 & $-0.34 * *$ & -0.19 & $-0.47 * *$ & -0.09 \\
\hline Male & 0.50 & $-1.25^{*}$ & -0.32 & -0.56 & 0.12 & -1.11 \\
\hline \multicolumn{7}{|l|}{ Region $^{c}$} \\
\hline West & Ref & Ref & Ref & Ref & Ref & Ref \\
\hline North & -0.22 & -0.19 & -0.50 & -1.18 & -0.97 & -0.52 \\
\hline East & -0.88 & -0.07 & 0.32 & 0.21 & -0.17 & 0.38 \\
\hline South & -0.78 & -0.32 & -0.03 & -0.69 & -0.74 & -0.94 \\
\hline Foreign country of birth parents & -0.33 & -0.60 & 0.45 & 0.32 & 0.37 & $1.95 *$ \\
\hline \multicolumn{7}{|l|}{ Educational level parents ${ }^{\mathrm{d}}$} \\
\hline Low & Ref & Ref & Ref & Ref & Ref & Ref \\
\hline Intermediate & -0.68 & 0.78 & 1.60 & $2.24 *$ & $2.30 *$ & 1.19 \\
\hline High & 0.87 & 1.51 & 1.12 & 1.94 & $2.10^{*}$ & 0.02 \\
\hline Single parent family & $-3.00 * *$ & -0.76 & $1.46^{*}$ & 1.02 & 0.91 & $2.07 * *$ \\
\hline \multicolumn{7}{|l|}{ Number of children in family } \\
\hline 1 Child & Ref & Ref & Ref & Ref & Ref & Ref \\
\hline 2 Children & 0.37 & -0.81 & 0.31 & 0.08 & 1.29 & 0.11 \\
\hline 3 Or more children & 0.60 & 0.16 & -0.14 & -0.17 & $2.07 * *$ & 1.26 \\
\hline Negative change in work situation & -0.88 & -0.46 & $3.01 * *$ & $2.45^{* *}$ & $1.71 * *$ & $2.53 * *$ \\
\hline Infected relative/friend with COVID-19 & 0.96 & 0.84 & $1.94 * *$ & 1.11 & 0.11 & 0.99 \\
\hline Daycare/school attendance child & -0.65 & 0.20 & 1.20 & 2.04 & 1.15 & 2.73 \\
\hline
\end{tabular}

$B$ unstandardized regression coefficient of multivariable linear regression model

$* * P$ value $<0.01, * P$ value $<0.05$

${ }^{a}$ Higher scores indicate better functioning

${ }^{\mathrm{b}}$ Higher scores indicate more symptoms

${ }^{\mathrm{c}}$ Region: North=Groningen, Friesland, Drenthe, East=Overijssel, Gelderland, Flevoland, South=Zeeland, Noord-Brabant, Limburg, West $=$ Utrecht, Noord-Holland, Zuid-Holland

${ }^{\mathrm{d}}$ Educational level parents divided into three categories: Low = primary, lower vocational education, lower and middle general secondary education; Intermediate $=$ middle vocational education, higher secondary education, pre-university education; High = higher vocational education, university

\section{Discussion}

This study compared the mental and social health of a representative sample of children and adolescents from the general population during the COVID-19 lockdown to a similar sample of children and adolescents before COVID-19. Children and adolescents reported poorer mental and social health during the COVID-19 lockdown on all six PROMIS domains. Substantial differences in percentages of children reporting severe anxiety and sleep-related impairment were observed. Fewer children and adolescents reported a poor global health during the COVID-19 lockdown, although the mean global health score was lower in this sample as compared to the sample before COVID-19. Significant associations with mental and social health complaints during the COVID-19 lockdown were found for family composition (growing up in a single-parent family or having three or more children in the family), a negative change in work situation of parents due to COVID-19 regulations, and an infected relative/friend with COVID-19. Children and adolescents reported a small decrease in the atmosphere at home during the lockdown. The majority of children and adolescents revealed a negative impact of the COVID-19 regulations on their daily life, that far outweigh the number of children who reported a positive effect. Especially missing contact with friends was considered important.

The results of this study confirm the suspicions of child and youth care professionals that the COVID-19 lockdown has negative effects on the mental and social health of children and adolescents. In opinion papers, professionals elaborated on the vulnerability of this group and expected more feelings of loneliness, anxiety and depression, as well as a more tense atmosphere at home [11-14]. Concerns were also expressed that the COVID-19 lockdown would lead to an increase in inequality and that children and families with lower socioeconomic status would be more susceptible to 
Table 5 Themes regarding the negative impact of COVID-19 regulations ranked according to their frequency of occurrence (high to low)

\begin{tabular}{ll}
\hline Themes & Illustrative quotes as reaction to the question 'How are the corona-regulations for you'? \\
\hline $\begin{array}{l}\text { 1. Missing contact with friends } \\
\text { 2. Not allowed to go to school }\end{array}$ & $\begin{array}{l}\text { 'I miss my friends' } \\
\text { 'Feeling alone, because I cannot see my friends anymore, it is different online' } \\
\text { 'It is a pity that I cannot go to school' } \\
\text { 'I miss my teacher and my class' } \\
\text { 'I am only allowed to play in and around the house' } \\
\text { 'I have to stay at home, I am not allowed to go anywhere' } \\
\text { 'I used to play soccer three times a week, I miss playing sports and being outside the most' } \\
\text { 'I am not allowed to play field hockey anymore' } \\
\text { 'I want to party with my friends, to participate in my final exams and I want to go on a holi- } \\
\text { day but that is not possible now' } \\
\text { 'It was my birthday and I was sad that nobody could visit me' } \\
\text { 'It is not possible to go shopping or do fun things' } \\
\text { 'I miss my daily routine and the boundary between school and home is completely gone' } \\
\text { 'Schoolwork at home is not easy, I can concentrate better at school' } \\
\text { 'I have the feeling that I do not learn anything, because homework is not discussed or revised' } \\
\text { 'I am not allowed to see or hug my grandparents' } \\
\text { 'I miss my extended family a lot' } \\
\text { 'I am often bored, because we cannot do anything' } \\
\text { 'It is boring to play alone all the time' }\end{array}$ \\
7. Missing extended family &
\end{tabular}

mental health issues $[12,15,16]$. Although this study could not definitely confirm these concerns, children from single parent families, from families with three or more children, and with parents who had a negative change in work situation reported more mental and social health problems during the COVID-19 lockdown.

While the Dutch partial lockdown was substantially different from the Chinese full lockdown, our results are in line with the three studies from China [17-19], who also reported higher anxiety and depressive symptom during the COVID-19 lockdown. Likewise, one of the Chinese studies also found that having an infected relative/friend with COVID-19 was predictive of more anxiety [19]. Germany had comparable restrictions and also found worse mental health and higher levels of anxiety in children and adolescents during lockdown [20]. In addition to the effects on anxiety and depressive symptoms, our study results show negative effects of the COVID-19 lockdown on anger, sleeprelated impairment and peer relationships.

Although the mean $T$-score on Global Health was lower (worse) in the sample during the lockdown as compared to before COVID-19, a lower percentage of children and adolescents reported poor Global Health during COVID-19. As data collection procedures were identical and the same measures were administered, methodological reasons are unlikely to cause these differences. There are a few possible explanations for this finding. It may be explained by the fact that the cut-offs are population-based and may not accurately represent clinically relevant cases as they have not been researched extensively. Secondly, cross-sectional data collection resulted in two independent samples, which may have had differences in the baseline distributions of clinical scores. Examining the item responses on the Global Health scale items indicated that less children reported "Poor" functioning on all items during COVID-19 (resulting in less clinical scores). However, the response category "Excellent" was also used less frequently (resulting in lower mean scores), which may have been a result of the limitations on daily life caused by the COVID-19 restrictions. Given the above possible explanations, this finding remains puzzling and prompts further study in a longitudinal design that is underway in our department.

Some limitations of this study need to be taken into account. First of all, self-reported responses may be influenced by social desirability. However, as the PROMIS data collection process was the same for the 2018 and 2020 sample we think it is unlikely that social desirability accounts for the differences observed between these samples. Secondly, although the aim was to obtain two representative samples that were comparable, significant differences with small effect sizes were found on age, parental educational level, and family composition. Parental country of birth did show a large risk ratio. As matching was not possible due to missing values in background characteristics in one of the samples, these differences between groups were corrected for by means of ANCOVAs. For comparing Anxiety and Depressive Symptoms, we were unable to correct for parental educational level in the ANCOVA, as this variable was not usable due to different categorization in the before COVID-19 sample. It is unlikely this influenced the results, as the effect size of this variable was small on other domains (Cramer's $V$ 
below 0.1). In addition, the data collection during COVID19 took place in April and May (2020), whereas the study data collection before COVID on anxiety and depression mainly took place in January and February (2018). Worse mental health is often reported during winter times [33]. This difference could have led to an underestimation of the actual impact of the COVID-19 lockdown.

We found that children and adolescents from families with certain risk factors (e.g. single-parent families) are more vulnerable to mental and social health problems. These children and adolescents should be in sight of health care professionals. However, in this study children and adolescents with existing mental or somatic problems were not included, while it is conceivable that these groups are even more vulnerable. More research is needed to study the mental and social health of these groups as well as to gain insight into the longitudinal effects, and to clarify if lower mental and social health scores are mainly due to the COVID-19 pandemic or the lockdown.

During the finalization of this paper, the Netherlands, and many other countries, are facing a second COVID-19 wave. This study showed that children and adolescents reported poorer mental and social health during the COVID-19 lockdown compared to before and exposed several risk factors for poor mental and social health. These risk factors and the effect of governmental regulations regarding lockdown on the mental and social health of children and adolescents should be taken into consideration when imposing new governmental regulations and should thus be brought to the forefront of political decision making and mental health care policy, intervention, and prevention.

Acknowledgements We would like to acknowledge Stichting Steun Emma Kinderziekenhuis, the Dutch National Health Care Institute, and the Netherlands Organization for Health Research and Development for their funding. Additionally, we would like to thank all children and adolescents that completed the questionnaires and Biomedia and Panel Inzicht for their support in setting up the research website and sending out the questionnaires.

Author contributions LH conceived the study. MAJL, MMvM, LT, HAvO, and LH conceptualized and designed the study. MAJL, MMvM, LT, HAvO, CBT and LH developed the sociodemographic questionnaire and MAJL, MMvM and LT carried out the data collection. MMvM, MAJL, LT and JZ performed the statistical analyses and $\mathrm{HAvO}$ and LT performed the qualitative analyses. CBT supervised the statistical analyses. The first draft of the manuscript was written by MMvM, MAJL and LH, and all authors commented on previous versions of the manuscript. LH, HAvO, TJCP, AP, KJO and CBT supervised the process. All authors critically revised the manuscript for intellectual content and approved the final version. MAJL and MMvM contributed equally as co-first authors.

Funding Data collection in this study was supported by Stichting Steun Emma Kinderziekenhuis, the Dutch National Health Care Institute, and the Netherlands Organization for Health Research and Development.
Data availability Data are available upon reasonable request.

Code availability Code is available upon reasonable request.

\section{Compliance with ethical standards}

Conflicts of interest All authors declare that they have no conflict of interest.

Ethical approval The Medical Ethical Committee of the Amsterdam UMC (location AMC and VUMC) approved the protocol and judged that the Dutch Medical Research Involving Human Subjects Act does not apply to this study. All procedures performed were in accordance with the 1964 Helsinki declaration and its later amendments or comparable ethical standards.

Informed consent Informed consent was obtained from all individual participants and/or legal guardians included in this study.

Open Access This article is licensed under a Creative Commons Attribution 4.0 International License, which permits use, sharing, adaptation, distribution and reproduction in any medium or format, as long as you give appropriate credit to the original author(s) and the source, provide a link to the Creative Commons licence, and indicate if changes were made. The images or other third party material in this article are included in the article's Creative Commons licence, unless indicated otherwise in a credit line to the material. If material is not included in the article's Creative Commons licence and your intended use is not permitted by statutory regulation or exceeds the permitted use, you will need to obtain permission directly from the copyright holder. To view a copy of this licence, visit http://creativecommons.org/licenses/by/4.0/.

\section{References}

1. Shen, K.-L., Yang, Y.-H., Jiang, R.-M., Wang, T.-Y., Zhao, D.-C., Jiang, Y., et al. (2020). Updated diagnosis, treatment and prevention of COVID-19 in children: Experts' consensus statement (condensed version of the second edition). World Journal of Pediatrics, 16(3), 232-239. https://doi.org/10.1007/s12519-020-00362-4

2. Fong, V. C., \& Iarocci, G. (2020). Child and family outcomes following pandemics: A systematic review and recommendations on COVID-19 policies. Journal of Pediatric Psychology. https://doi. org/10.1093/jpepsy/jsaa092

3. Kwong, A. S. F., Pearson, R. M., Adams, M. J., Northstone, K., Tilling, K., Smith, D., et al. (2020). Mental health during the COVID-19 pandemic in two longitudinal UK population cohorts. medRxiv. https://doi.org/10.1101/2020.06.16.20133116.

4. Wang, C., Pan, R., Wan, X., Tan, Y., Xu, L., Ho, C. S., et al. (2020). Immediate psychological responses and associated factors during the initial stage of the 2019 coronavirus disease (COVID19) epidemic among the general population in China. International Journal of Environmental Research and Public Health, 17(5), 1729. https://doi.org/10.3390/ijerph17051729

5. Gualano, M. R., Lo Moro, G., Voglino, G., Bert, F., \& Siliquini, R. (2020). Effects of COVID-19 lockdown on mental health and sleep disturbances in Italy. International Journal of Environmental Research and Public Health, 17(13), 4779. https://doi.org/10. 3390/ijerph17134779

6. Vindegaard, N., \& Benros, M. E. (2020). COVID-19 pandemic and mental health consequences: Systematic review of the current evidence. Brain, Behavior, and Immunity. https://doi.org/10. 1016/j.bbi.2020.05.048 
7. O'Connor, R. C., Wetherall, K., Cleare, S., McClelland, H., Melson, A. J., Niedzwiedz, C. L., et al. (2020). Mental health and wellbeing during the COVID-19 pandemic: Longitudinal analyses of adults in the UK COVID-19 Mental Health \& Wellbeing study. British Journal of Psychiatry. https://doi.org/10.1192/bjp.2020. 212

8. Pierce, M., Hope, H., Ford, T., Hatch, S., Hotopf, M., John, A., et al. (2020). Mental health before and during the COVID-19 pandemic: A longitudinal probability sample survey of the UK population. The Lancet Psychiatry, 7(10), 883-892. https://doi. org/10.1016/S2215-0366(20)30308-4

9. Pieh, C., Budimir, S., \& Probst, T. (2020). The effect of age, gender, income, work, and physical activity on mental health during coronavirus disease (COVID-19) lockdown in Austria. Journal of Psychosomatic Research, 136, 110186. https://doi.org/10.1016/j. jpsychores.2020.110186

10. Wang, C., Pan, R., Wan, X., Tan, Y., Xu, L., McIntyre, R. S., et al. (2020). A longitudinal study on the mental health of general population during the COVID-19 epidemic in China. Brain, Behavior, and Immunity. https://doi.org/10.1016/j.bbi.2020.04.028

11. Loades, M. E., Chatburn, E., Higson-Sweeney, N., Reynolds, S., Shafran, R., Brigden, A., et al. (2020). Rapid systematic review: The impact of social isolation and loneliness on the mental health of children and adolescents in the context of COVID-19. Journal of the American Academy of Child and Adolescent Psychiatry. https://doi.org/10.1016/j.jaac.2020.05.009

12. Fegert, J. M., Vitiello, B., Plener, P. L., \& Clemens, V. (2020). Challenges and burden of the Coronavirus 2019 (COVID-19) pandemic for child and adolescent mental health: A narrative review to highlight clinical and research needs in the acute phase and the long return to normality. Child and Adolescent Psychiatry and Mental Health, 14, 1-11. https://doi.org/10.1186/ s13034-020-00329-3

13. Galea, S., Merchant, R. M., \& Lurie, N. (2020). The mental health consequences of COVID-19 and physical distancing: The need for prevention and early intervention. JAMA Internal Medicine, 180(6), 817-818. https://doi.org/10.1001/jamainternmed.2020. 1562

14. Green, P. (2020). Risks to children and young people during covid-19 pandemic. BMJ, 369, m1669. https://doi.org/10.1136/ bmj.m1669

15. Valenzuela, J., Crosby, L. E., \& Harrison, R. R. (2020). Commentary: Reflections on the COVID-19 pandemic and health disparities in pediatric psychology. Journal of Pediatric Psychology, 45(8), 839-841. https://doi.org/10.1093/jpepsy/jsaa063

16. Golberstein, E., Wen, H., \& Miller, B. F. (2020). Coronavirus disease 2019 (COVID-19) and mental health for children and adolescents. JAMA Pediatrics. https://doi.org/10.1001/jamapediatrics. 2020.1456

17. Xie, X., Xue, Q., Zhou, Y., Zhu, K., Liu, Q., Zhang, J., et al. (2020). Mental health status among children in home confinement during the coronavirus disease 2019 outbreak in Hubei Province, China. JAMA Pediatrics. https://doi.org/10.1001/jamapediatrics. 2020.1619

18. Zhou, S.-J., Zhang, L.-G., Wang, L.-L., Guo, Z.-C., Wang, J.-Q., Chen, J.-C., et al. (2020). Prevalence and socio-demographic correlates of psychological health problems in Chinese adolescents during the outbreak of COVID-19. European Child \& Adolescent Psychiatry. https://doi.org/10.1007/s00787-020-01541-4

19. Duan, L., Shao, X., Wang, Y., Huang, Y., Miao, J., Yang, X., et al. (2020). An investigation of mental health status of children and adolescents in china during the outbreak of COVID-19. Journal of Affective Disorders, 275, 112-118. https://doi.org/10.1016/j. jad.2020.06.029

20. Ravens-Sieberer, U., Kaman, A., Erhart, M., Devine, J., Schlack, R., \& Otto, C. (2021). Impact of the COVID-19 pandemic on quality of life and mental health in children and adolescents in Germany. European Child \& Adolescent Psychiatry. https://doi. org/10.1007/s00787-021-01726-5

21. Bignardi, G., Dalmaijer, E. S., Anwyl-Irvine, A. L., Smith, T. A., Siugzdaite, R., Uh, S., et al. (2020). Longitudinal increases in childhood depression symptoms during the COVID-19 lockdown. Archives of Disease in Childhood. https://doi.org/10.1136/archd ischild-2020-320372

22. Magson, N. R., Freeman, J. Y. A., Rapee, R. M., Richardson, C. E., Oar, E. L., \& Fardouly, J. (2021). Risk and protective factors for prospective changes in adolescent mental health during the COVID-19 pandemic. Journal of Youth and Adolescence, 50(1), 44-57. https://doi.org/10.1007/s10964-020-01332-9

23. Hawes, M. T., Szenczy, A. K., Klein, D. N., Hajcak, G., \& Nelson, B. D. (2021). Increases in depression and anxiety symptoms in adolescents and young adults during the COVID-19 pandemic. Psychological Medicine. https://doi.org/10.1017/s0033291720005358

24. Luijten, M. A., van Litsenburg, R. R., Terwee, C. B., Grootenhuis, M. A., \& Haverman, L. (2021). Psychometric properties of the Patient-Reported Outcomes Measurement Information System (PROMIS $®$ ) pediatric item bank peer relationships in the Dutch general population. Quality of Life Research. https://doi.org/10. 1002/acr.24094

25. Haverman, L., van Oers, H. A., Limperg, P. F., Hijmans, C. T., Schepers, S. A., Sint Nicolaas, S. M., et al. (2014). Implementation of electronic patient reported outcomes in pediatric daily clinical practice: The KLIK experience. Clinical Practice in Pediatric Psychology, 2(1), 50-67. https://doi.org/10.1037/cpp0000043

26. Cella, D., Gershon, R., Lai, J. S., \& Choi, S. (2007). The future of outcomes measurement: Item banking, tailored short-forms, and computerized adaptive assessment. QualLife Research, 16 (Suppl 1), 133-141. https://doi.org/10.1007/s11136-007-9204-6

27. Irwin, D. E., Stucky, B. D., Langer, M. M., Thissen, D., DeWitt, E. M., Lai, J. S., et al. (2012). PROMIS Pediatric Anger Scale: An item response theory analysis. Quality of Life Research, 21(4), 697-706. https://doi.org/10.1007/s11136-011-9969-5

28. DeWalt, D. A., Thissen, D., Stucky, B. D., Langer, M. M., Morgan, D. E., Irwin, D. E., et al. (2013). PROMIS Pediatric Peer Relationships Scale: Development of a peer relationships item bank as part of social health measurement. Health Psychology, 32(10), 1093-1103. https://doi.org/10.1037/a0032670

29. Forrest, C. B., Bevans, K. B., Pratiwadi, R., Moon, J., Teneralli, R. E., Minton, J. M., et al. (2014). Development of the PROMIS (R) pediatric global health (PGH-7) measure. QualLife Research, 23(4), 1221-1231. https://doi.org/10.1007/s11136-013-0581-8

30. Forrest, C. B., Meltzer, L. J., Marcus, C. L., de la Motte, A., Kratchman, A., Buysse, D. J., et al. (2018). Development and validation of the PROMIS Pediatric Sleep Disturbance and SleepRelated Impairment item banks. Sleep. https://doi.org/10.1093/ sleep/zsy054

31. Irwin, D., Stucky, B., Langer, M., Thissen, D., DeWitt, E., Lai, J. S., et al. (2010). An item response analysis of the pediatric PROMIS anxiety and depressive symptoms scales. Quality of Life Research, 19(4), 595-607. https://doi.org/10.1007/ s11136-010-9619-3

32. Braun, V., \& Clarke, V. (2006). Using thematic analysis in psychology. Qualitative Research in Psychology, 3(2), 77-101. https://doi.org/10.1191/1478088706qp063oa

33. Jia, H., \& Lubetkin, E. I. (2009). Time trends and seasonal patterns of health-related quality of life among US adults. Public Health Reports, 124(5), 692-701. https://doi.org/10.1177/00333 5490912400511

Publisher's Note Springer Nature remains neutral with regard to jurisdictional claims in published maps and institutional affiliations. 